\title{
Treatment Outcomes at Skeletal Maturity after Calcaneo-Cuboid-Cuneiform Osteotomy for Symptomatic Flatfoot Deformity in Children
}

\author{
Jung Ryul Kim, MD, Ki Bum Kim, MD, Seong Woo Chong, MD, \\ Dong Hun Ham, MD*, Sung Il Wang, MD
}

Department of Orthopaedic Surgery, Jeonbuk National University Medical School, Research Institute for Endocrine Sciences and Research Institute of Clinical Medicine of Jeonbuk National University-Biomedical Research Institute of Jeonbuk National University Hospital, Jeonju,

${ }^{*}$ Department of Orthopaedic Surgery, St. Carollo Hospital, Suncheon, Korea

Background: The purpose of this study was to evaluate clinical and radiological outcomes at skeletal maturity after a calcaneocuboid-cuneiform osteotomy (triple C osteotomy) for symptomatic flatfoot deformity compared with healthy young adult controls. Methods: Nineteen patients (30 feet) who undergone a triple $\mathrm{C}$ osteotomy for idiopathic symptomatic flatfeet from July 2006 to April 2013 were compared with 19 controls (38 feet). Radiographic measurements at preoperative examination, 1-year postoperative follow-up, and follow-up at skeletal maturity were evaluated. Functional outcomes were assessed by using the validated visual analog scale foot and ankle (VAS-FA) and the modified American Orthopaedic Foot and Ankle Surgery (AOFAS) score.

Results: In the triple $\mathrm{C}$ osteotomy group, 11 of 12 radiographic measurements were significantly improved at 1 year postoperatively and the last follow-up $(p<0.001)$. There was no recurrence at skeletal maturity $(p>0.05)$. There were no significant differences in nine of 12 radiographic measurements between the triple $\mathrm{C}$ osteotomy group at maturity and the control group ( $p>0.05)$. Average VAS-FA and AOFAS scores were significantly improved at the time of skeletal maturity $(p<0.001)$.

Conclusions: Surgical correction of symptomatic flatfoot deformity in childhood resulted in favorable outcomes after the triple $\mathrm{C}$ osteotomy. Deformity correction was also maintained during follow-up at skeletal maturity.

Keywords: Flatfoot, Children, Calcaneus, Cuboid, Cuneiform, Osteotomy

Most cases of flexible pes planovalgus in children can resolve spontaneously during the development of the medial longitudinal arch of the foot with increasing age. ${ }^{1-3)}$ The main treatment of recalcitrant flexible pes planovalgus involves conservative measures including shoe modifications and stretching exercises. ${ }^{2)}$ Nevertheless, surgical treatment

Received April 28, 2019; Accepted December 24, 2019

Correspondence to: Sung II Wang, MD

Department of Orthopaedic Surgery, Jeonbuk National University Medical School, Research Institute for Endocrine Sciences and Research Institute of Clinical Medicine of Jeonbuk National University-Biomedical Research Institute of Jeonbuk National University Hospital, 567 Baekje-daero, Deokjin-gu, Jeonju 54896, Korea

Tel: +82-63-250-1760, Fax: +82-63-271-6538

E-mail:wsi1205@naver.com may be needed when prolonged conservative treatment fails to relieve symptoms. A number of procedures including soft-tissue reconstruction and bone modifications have been described as surgical options to correct flatfoot deformities. Each procedure has its own advantages and disadvantages with varying results. ${ }^{4-7)}$ The medial calcaneal sliding-opening wedge cuboid-closing wedge cuneiform osteotomy (also known as a triple C osteotomy), first described by Rathjen and Mubarak, ${ }^{8)}$ is one of such procedures. The triple $\mathrm{C}$ osteotomy is advantageous for obtaining correction at the position of each flatfoot deformity. Medial calcaneal sliding provides correction of the valgus hindfoot and medial shifting of the mechanical pull of the tendon, thus eliminating the negative effect of the Achilles tendon on flatfoot deformity. The cuboid opening wedge osteotomy can lengthen the lateral column and realign

Copyright (C) 2020 by The Korean Orthopaedic Association 
the talonavicular joint. The cuneiform plantar closing wedge osteotomy allows pronation and plantar flexion of the forefoot and restores the normal talar-first metatarsal (MTT) angle. This technique has been associated with good results in the treatment of severe deformity with neurological conditions as well as symptomatic idiopathic flexible flatfeet in a pediatric population., ${ }^{9,10)}$

However, there has been no published study regarding the mid- or long-term outcome of this procedure in the symptomatic flatfoot. We hypothesized that a triple $\mathrm{C}$ osteotomy would allow favorable outcomes with maintenance of deformity correction during follow-up at skeletal maturity. Thus, the purpose of this study was to evaluate clinical and radiological outcomes at skeletal maturity following triple $\mathrm{C}$ osteotomy for symptomatic flatfoot deformity compared with healthy young adult controls.

\section{METHODS}

We conducted this study in compliance with the principles of the Declaration of Helsinki. The design and protocol of this retrospective case-control study were approved by the Institutional Review Board of Jeonbuk University Hospital (IRB No. 2020-03-025). Informed consent was waived since this study was conducted retrospectively. Flatfoot deformity was diagnosed on the basis of weight-bearing radiographs showing formation of the medial arch and a valgus hindfoot (Fig. 1A and B). Severe deformity was de- fined as a varus position of the heel on tiptoe in those who could not reconstitute the arch. Inclusion criteria were as follows: (1) consecutive patients who underwent a triple C procedure due to failure of prolonged conservative treatment for recalcitrant idiopathic flatfoot deformity between July 2006 and April 2013 and (2) patients with weightbearing anteroposterior (AP) and lateral radiographs of the foot obtained preoperatively and postoperatively until skeletal maturity (Risser 5). Patients were excluded if they (1) had underlying neurological or syndromic conditions or tarsal coalition, (2) had no additional procedure except peroneus brevis lengthening or tendo-Achilles lengthening, (3) had inadequate radiographs for measurement, or (4) did not reach skeletal maturity. During the study period, 22 patients ( 35 feet) who were diagnosed as having idiopathic severe flatfeet underwent a triple $\mathrm{C}$ procedure. Of them, three patients (five feet) were lost to followup. Thus, 19 patients (30 feet, triple C group) who were available for follow-up were included in this study. The mean age at the time of assessment was 19.5 years (range, $17-23.8$ years). The mean age at the time of operation was 12.5 years (range, $9-15$ years). The mean follow-up period was 6.7 years (range, 3.9-9.8 years). Nineteen age- and sex-matched healthy young adults (15 men and four women) were also evaluated. None of these control subjects had any activity-restricting musculoskeletal condition or history of orthopedic treatment. The mean age of the control group was 19.6 years (range, 15-26 years) (Table 1).
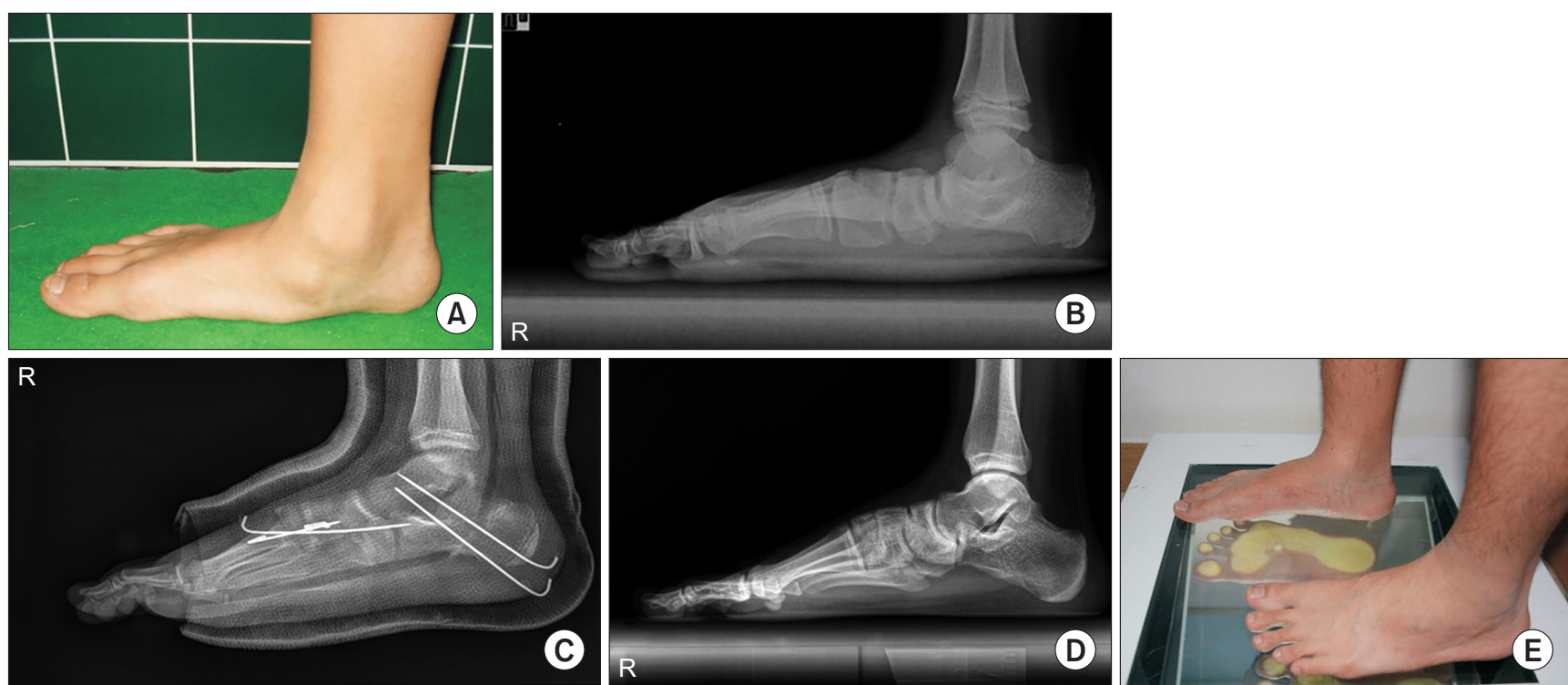

Fig. 1. Triple $C$ osteotomy in a 11-year-old boy with symptomatic flatfoot deformity. (A) Preoperative photograph. (B) Weight-bearing lateral radiograph. (C) Intraoperative lateral radiograph during the triple $\mathrm{C}$ osteotomy. The weight-bearing lateral radiograph (D) and podoscope image (E) taken at 6 years after operation, showing improvement of the foot alignment. 
Kim et al. Calcaneo-Cuboid-Cuneiform Osteotomy for Symptomatic Flatfoot Deformity in Children

Clinics in Orthopedic Surgery • Vol. 12, No. 2, $2020 \bullet$ www.ecios.org

\begin{tabular}{|c|c|c|c|}
\hline Variable & $\begin{array}{l}\text { Normal control } \\
\quad(n=19)\end{array}$ & $\begin{array}{l}\text { Triple C } \\
(n=19)\end{array}$ & $p$-value \\
\hline Sex & & & 1.00 \\
\hline Male & 15 & 15 & \\
\hline Female & 4 & 4 & \\
\hline Follow-up (yr) & - & $6.7 \pm 1.2$ & - \\
\hline Age at the time of assessment (yr) & $19.6 \pm 2.4$ & $19.2 \pm 2.1$ & 0.57 \\
\hline Body mass index $\left(\mathrm{kg} / \mathrm{m}^{2}\right)$ & $22.2 \pm 1.8$ & $22.8 \pm 1.5$ & 0.63 \\
\hline
\end{tabular}

Values are presented as mean \pm standard deviation. Unpaired $t$-test was used to evaluate the statistical significance of demographic data between normal controls and triple $\mathrm{C}$ group patients.

\section{Surgical Technique}

All procedures were performed by a single surgeon (JRK) (Fig. 1C). Surgical techniques used in this study were basically the same as those reported by Rathjen and Mubarak. ${ }^{8)}$ In addition to an isolated bony procedure, soft-tissue procedures were performed concomitantly in some patients: peroneus brevis lengthening in nine feet and tendoAchilles lengthening in 15 feet. A Z-plasty of the peroneus brevis was performed at the musculotendinous junction when there was a tight peroneus brevis after medial calcaneal sliding osteotomy. A tendo-Achilles lengthening was performed when the dorsiflexion of the ankle was less than $10^{\circ}$ with the knee extended. ${ }^{11)}$

\section{Radiologic Measurements}

The relative alignment of each segment of the foot was assessed by using weight-bearing AP and lateral radiographs taken preoperatively, 1 year postoperatively, and at skeletal maturity in all patients (Fig. 1D). Twelve radiologic measurements were assessed as described by Davids et al. ${ }^{9)}$ and Moraleda and Mubarak. ${ }^{12)}$ Four radiologic measurements-talo-first MTT angle, talonavicular coverage, talocalcaneal angle, and talo-fifth MTT angle-were evaluated by using weight-bearing AP radiographs. Weight-bearing lateral radiographs were used to calculate the following eight measurements: talo-first MTT angle, calcaneal pitch, calcaneo-fifth MTT angle, talocalcaneal angle, talohorizontal angle, first-fifth MTT angle, naviculo-cuboid overlap, and medial-lateral column ratio. For the medial-lateral column ratio, the medial column length ranged from the distal margin of the head of the great toe MTT to the most proximal margin of the posterior dome of the talus. The lateral column length ranged from the distal most margin of the fifth toe MTT to the most proximal margin of the posterior aspect of the calcaneus.9) Three orthopedic surgeons (KBK, SWC, DHH) evaluated the interobserver reliability of radiologic measurements. Intraclass correlation coefficient (ICC) and their 95\% confidence interval (CI) were evaluated: an ICC less than 0.00 , poor reliability; 0.00 to 0.20 , slight reliability; 0.21 to 0.40 , fair reliability; 0.41 to 0.60 , moderate reliability; 0.61 to 0.80 , substantial agreement; and 0.81 to 1.00 , excellent or almost perfect agreement.

\section{Clinical Evaluations}

Clinical outcomes were assessed with the validated visual analog scale for foot and ankle (VAS-FA) and the American Orthopaedic Foot and Ankle Surgery (AOFAS) score for both hindfoot and midfoot sections. These scores all have a maximum value of 100 points. ${ }^{13,14)}$ The flatfoot module of the AOFAS ankle-midfoot score system consists of three components: pain (40 points), function (45 point), and alignment (15 points). The AOFAS ankle-hindfoot score system consists of pain (40 points), function (40 points), and alignment (10 points). A podoscope examination was performed in an objective and reliable manner to demonstrate the weight-bearing profile of the foot (Fig. 1E).

\section{Statistical Analysis}

Unpaired $t$-test was used to evaluate statistical significance in difference of demographic data between normal controls and patients in the triple $\mathrm{C}$ group. Paired $t$-test was used to evaluate statistical significance of difference between preoperative and postoperative radiographic or functional results. All statistical analyses were performed by using SPSS ver. 18.0 (SPSS Inc., Chicago, IL, USA). Statistical significance was considered when $p$-value was less than 0.05 .

\section{RESULTS}

\section{Participant Characteristics}

Basic anthropometric parameters such as sex, age, and body mass index were similar between patients of the triple $\mathrm{C}$ group and normal controls (Table 1).

\section{Radiographic Results}

All radiographic measurements showed excellent interobserver reliability (ICC, 0.81-0.96) (Table 2). Comparison of preoperative and 1-year postoperative follow-up weightbearing radiographs showed significant improvement in 11 of 12 radiographic measurements except for the mediallateral column ratio. Correction of preoperative deformity 
Kim et al. Calcaneo-Cuboid-Cuneiform Osteotomy for Symptomatic Flatfoot Deformity in Children

Clinics in Orthopedic Surgery • Vol. 12, No. 2, $2020 \bullet$ www.ecios.org

Table 2. Interobserver Reliability of Radiographic Measurement

\begin{tabular}{|ll}
\hline \multicolumn{1}{|c}{ Radiographic measurement } & ICC (95\% CI) \\
\hline AP talo-first MTT angle $\left(^{\circ}\right)$ & $0.87(0.73-0.94)$ \\
\hline AP talonavicular coverage angle $\left(^{\circ}\right)$ & $0.92(0.86-0.96)$ \\
\hline AP talocalcaneal angle $\left(^{\circ}\right)$ & $0.89(0.79-0.94)$ \\
\hline AP talo-fifth MTT angle $\left(^{\circ}\right)$ & $0.90(0.81-0.95)$ \\
\hline Lateral talo-first MTT angle $\left(^{\circ}\right)$ & $0.89(0.81-0.94)$ \\
\hline Lateral calcaneal pitch angle $\left(^{\circ}\right)$ & $0.85(0.72-0.92)$ \\
\hline Lateral calcaneo-fifth MTT angle $\left(^{\circ}\right)$ & $0.86(0.75-0.92)$ \\
\hline Lateral talocalcaneal angle $\left(^{\circ}\right)$ & $0.91(0.82-0.95)$ \\
\hline Lateral talohorizontal angle $\left(^{\circ}\right)$ & $0.92(0.84-0.96)$ \\
\hline Lateral first-fifth MTT angle $\left(^{\circ}\right)$ & $0.89(0.80-0.94)$ \\
\hline Lateral naviculo-cuboid overlap $(\%)$ & $0.81(0.63-0.89)$ \\
\hline Medial-lateral column ratio $(\%)$ & $0.96(0.90-0.98)$ \\
\hline
\end{tabular}

ICCs and 95\% Cls were used to summarize the interobserver reliability of radiographic measurements. They were calculated in the setting using a two-way random effect model assuming a single measurement and absolute agreement.

ICC: intraclass correlation coefficient, $\mathrm{Cl}$ : confidence interval, AP: anteroposterior, MTT: metatarsal. in these 11 radiographic measurements was maintained from 1-year postoperative to the last follow-up at skeletal maturity.

The medial-lateral column ratio did not show significant difference among the three time points: $104.2 \%$ $\pm 3.3 \%$ preoperatively vs. $103.5 \% \pm 4.0 \%$ at 1 -year postoperatively vs. $103.6 \% \pm 4.1 \%$ at the last follow-up. Comparison of the last follow-up radiographs with the normal controls did not show significant difference in nine of 12 radiographic measurements except for the AP talo-first MTT angle, the AP talonavicular coverage angle, and the lateral calcaneo-fifth MTT angle (Table 3).

\section{Clinical Outcome Surveys}

A comparison of preoperative values and final follow-up functional outcomes showed significant improvement in the triple $\mathrm{C}$ group. Mean VAS-FA, AOFAS ankle-midfoot, and hindfoot scores were $32.6 \pm 7.3$ (range, 18-45), 39.9 \pm 4.3 (range, 29-49), and $40.6 \pm 4.6$ (range, 34-50), respectively, preoperatively and $89.6 \pm 6.4$ (range, 80-99), $87.5 \pm 9.5$ (range, 65-100), and $89.9 \pm 7.8$ (range, 67-100), respectively, at the last follow-up at skeletal maturity, indicating favorable outcomes (Table 4). Deformity correction was found maintained in the podoscope examination in all patients at the last follow-up at skeletal maturity.

Table 3. Radiological Results and Comparison among Preoperative, Postoperative 1-Year, and at Maturity Data

\begin{tabular}{|c|c|c|c|c|c|c|c|c|}
\hline Radiographic measurement & Preoperative & $\begin{array}{c}1-Y e a r \\
\text { postoperative }\end{array}$ & Maturity & $\begin{array}{l}\text { Normal } \\
\text { control }\end{array}$ & $\begin{array}{l}\text { Preoperative } \\
\text { vs. 1-year } \\
\text { postoperative* }\end{array}$ & $\begin{array}{l}\text { Preoperative } \\
\text { maturity* }\end{array}$ & $\begin{array}{l}\text { 1-Year } \\
\text { postoperative } \\
\text { maturity* }\end{array}$ & $\mathrm{Normal}^{+}{ }_{\text {maturity }}^{\dagger}$ \\
\hline AP talo-first MTT angle $\left({ }^{\circ}\right)$ & $25.3 \pm 6.8$ & $8.57 \pm 3.8$ & $8.4 \pm 3.1$ & $6.28 \pm 2.0$ & $<0.001$ & $<0.001$ & 0.73 & 0.017 \\
\hline AP talonavicular coverage angle $\left({ }^{\circ}\right)$ & $25.9 \pm 8.6$ & $9.42 \pm 3.1$ & $10.4 \pm 2.7$ & $8.40 \pm 2.5$ & $<0.001$ & $<0.001$ & 0.52 & 0.002 \\
\hline AP talocalcaneal angle $\left({ }^{\circ}\right)$ & $30.5 \pm 5.5$ & $21.2 \pm 6.7$ & $21.4 \pm 5.5$ & $22.3 \pm 4.7$ & $<0.001$ & $<0.001$ & 0.77 & 0.47 \\
\hline AP talo-fifth MTT angle $\left({ }^{\circ}\right)$ & $38.8 \pm 5.8$ & $31.5 \pm 5.5$ & $31.5 \pm 5.3$ & $30.4 \pm 4.5$ & $<0.001$ & $<0.001$ & 0.99 & 0.35 \\
\hline Lateral talo-first MTT angle $\left({ }^{\circ}\right)$ & $27.2 \pm 9.3$ & $7.32 \pm 2.7$ & $7.25 \pm 2.5$ & $6.30 \pm 2.6$ & $<0.001$ & $<0.001$ & 0.85 & 0.13 \\
\hline Lateral calcaneal pitch angle $\left({ }^{\circ}\right)$ & $6.32 \pm 3.5$ & $21.5 \pm 3.3$ & $22.1 \pm 2.9$ & $23.1 \pm 2.7$ & $<0.001$ & $<0.001$ & 0.11 & 0.14 \\
\hline Lateral calcaneo-fifth MTT angle $\left({ }^{\circ}\right)$ & $14.4 \pm 4.8$ & $26.6 \pm 5.0$ & $27.2 \pm 5.9$ & $32.7 \pm 5.0$ & $<0.001$ & $<0.001$ & 0.28 & $<0.001$ \\
\hline Lateral talocalcaneal angle $\left(^{\circ}\right)$ & $49.6 \pm 8.2$ & $39.2 \pm 3.7$ & $40.3 \pm 5.2$ & $41.8 \pm 3.6$ & $<0.001$ & $<0.001$ & 0.92 & 0.18 \\
\hline Lateral talohorizontal angle $\left({ }^{\circ}\right)$ & $42.3 \pm 9.9$ & $25.4 \pm 3.2$ & $25.4 \pm 3.6$ & $26.8 \pm 3.2$ & $<0.001$ & $<0.001$ & 0.96 & 0.09 \\
\hline Lateral fist-fifth MTT angle $\left(^{\circ}\right)$ & $9.3 \pm 3.5$ & $13.5 \pm 3.9$ & $14.2 \pm 4.7$ & $14.4 \pm 3.0$ & $<0.001$ & $<0.001$ & 0.13 & 0.88 \\
\hline Lateral naviculo-cuboid overlap (\%) & $78.4 \pm 9.6$ & $54.0 \pm 10$ & $53.7 \pm 10$ & $50.4 \pm 12$ & $<0.001$ & $<0.001$ & 0.83 & 0.24 \\
\hline Medial-lateral column ratio (\%) & $104.2 \pm 3.3$ & $103.5 \pm 4.0$ & $103.6 \pm 4.1$ & $103.8 \pm 2.2$ & 0.31 & 0.42 & 0.84 & 0.82 \\
\hline
\end{tabular}

Values are presented as mean \pm standard deviation.

AP: anteroposterior, MTT: metatarsal.

${ }^{*}$ Paired $t$-test. ${ }^{\dagger}$ Unpaired $t$-test between normal controls and triple $\mathrm{C}$ group patients at maturity. 


$\begin{aligned} & \text { Table 4. Functional Outcomes in Triple C Patients: VAS-FA Score and } \\
& \text { AOFAS Score }\end{aligned}$
\begin{tabular}{|cccr}
\multicolumn{1}{c}{ Variable } & Preoperative & Last follow-up & $p$-value \\
\hline VAS-FA score & $32.6 \pm 7.3$ & $89.6 \pm 6.4$ & $<0.001$ \\
\hline AOFAS midfoot score & $39.9 \pm 4.3$ & $87.5 \pm 9.5$ & $<0.001$ \\
\hline AOFAS hindfoot score & $40.6 \pm 4.6$ & $89.9 \pm 7.8$ & $<0.001$ \\
\hline
\end{tabular}

Values are presented as mean \pm standard deviation. Paired $t$-test was used to evaluate the statistical significance between preoperative and last follow-up functional outcomes in the triple $\mathrm{C}$ group.

VAS-FA: visual analog scale foot and ankle score, AOFAS: American Orthopaedic Foot and Ankle Society score.

\section{Complications}

One patient had mild signs of neurapraxia that successfully resolved within 2 months after the surgery. In one patient, there was talonavicular joint laxity and forefoot abduction (one feet), which could be considered midfoot arthrodesis. However, additional surgery was not performed because there was no pain. There were no wound complications or infections.

\section{DISCUSSION}

Our results show that foot function at maturity following a triple $\mathrm{C}$ osteotomy for recalcitrant idiopathic flatfoot deformity could be satisfactory. The pathoanatomy of idiopathic pes planovalgus is complex. It includes several key components. Our satisfactory outcome might be due to sufficient correction at the position of each flatfoot deformity after the triple $\mathrm{C}$ osteotomy. Many researchers have suggested that radiologic parameters for evaluating flatfoot deformity are closely related to clinical outcomes because they provide reliable information for each different part of flatfoot deformity. ${ }^{9,15,16)}$

In the current study, comparison of preoperative and 1-year postoperative follow-up weight-bearing radiographs showed significant improvement in 11 of 12 radiographic measurements (except the medial-lateral column ratio). Such improvement was maintained from 1-year postoperative to the last follow-up at skeletal maturity. Furthermore, eight of 11 radiographic measurements showed no significant difference from those of normal controls $(p>0.05)$.

In triple $\mathrm{C}$ osteotomy, a cuboid opening wedge osteotomy is performed to improve the relatively short lateral column in the planovalgus foot. In the present study, the medial-lateral column ratio showed no significant improvement from preoperative to the last follow-up at skeletal maturity $(p=0.42)$. However, since the medial-lateral column ratio at the last follow-up did not show a significant difference from that of the normal control group $(p=$ 0.82 ), the improvement of the short lateral column through triple $\mathrm{C}$ osteotomy requires further study on severe flatfoot deformity with more cases of the idiopathic flatfoot.

Meanwhile, we purposefully focused on patient-reported, subjective functional outcomes that were believed to be critical to evaluate effects of the surgery. We used VAS-FA and AOFAS score for both hindfoot and midfoot sections. ${ }^{13,14,16)}$ In the present study, the VAS-FA, AOFAS ankle-hindfoot, and midfoot scores were $32.6 \pm 7.3,39.9 \pm$ 4.3 , and $40.6 \pm 4.6$, respectively, preoperatively and $89.6 \pm$ $6.4,88.6 \pm 9.2$, and $91.4 \pm 7.6$, respectively, at the last follow-up at skeletal maturity, indicating favorable outcomes for both general and foot segment-related functions. Our study also confirmed the maintenance of corrected deformity and uniform weight load in the podoscope examination. On postoperative complications, there were no cases of wound infection. A sign of mild sural neurapraxia was found in one patient, which was resolved by the time of last follow-up. In one patient, there were talonavicular joint laxity and residual abduction of forefoot (one feet) that could require midfoot arthrodesis. However, because of the lack of pain, additional surgery was not performed. In physical examination, patients with abduction tendency of the forefoot and flatfoot deformity might need careful attention because these patients may not show improvement, or the alignment of the forefoot relative to the hindfoot can be exacerbated after a triple C osteotomy. Our study has some limitations. First, only a small number of patients were included in the study due to the retrospect design. Second, the inclusion of unilateral and bilateral cases in the same group could violate the principle of statistical independence. Third, selection bias might have occurred in the age- and sex-matched normal control group. Fourth, further research on osteoarthrosis that can occur secondary to a triple $\mathrm{C}$ osteotomy is needed. Nevertheless, the study population was homogeneous in terms of etiology of the condition. Our results demonstrated that the triple C procedure could correct severe idiopathic flatfoot deformity and that such correction could be maintained at skeletal maturity within normal limits. In conclusion, surgical correction of symptomatic flatfoot deformity in childhood can provide favorable outcomes after a triple $\mathrm{C}$ osteotomy, as well as maintenance of deformity correction during follow-up at skeletal maturity.

\section{CONFLICT OF INTEREST}

No potential conflict of interest relevant to this article was reported. 
Kim et al. Calcaneo-Cuboid-Cuneiform Osteotomy for Symptomatic Flatfoot Deformity in Children

Clinics in Orthopedic Surgery • Vol. 12, No. 2, $2020 \bullet$ www.ecios.org

\section{REFERENCES}

1. Staheli LT, Chew DE, Corbett M. The longitudinal arch: a survey of eight hundred and eighty-two feet in normal children and adults. J Bone Joint Surg Am. 1987;69(3):426-8.

2. Mosca VS. Flexible flatfoot in children and adolescents. J Child Orthop. 2010;4(2):107-21.

3. Kim HY, Shin HS, Ko JH, Cha YH, Ahn JH, Hwang JY. Gait analysis of symptomatic flatfoot in children: an observational study. Clin Orthop Surg. 2017;9(3):363-73.

4. Adelaar RS, Dannelly EA, Meunier PA, Stelling FH, Goldner JL, Colvard DF. A long term study of triple arthrodesis in children. Orthop Clin North Am. 1976;7(4):895-908.

5. Seymour N. The late results of naviculo-cuneiform fusion. J Bone Joint Surg Br. 1967;49(3):558-9.

6. Kuwada GT, Dockery GL. Complications following traumatic incidents with STA-peg procedures. J Foot Surg. 1988; 27(3):236-9.

7. Scher DM, Bansal M, Handler-Matasar S, Bohne WH, Green DW. Extensive implant reaction in failed subtalar joint arthroereisis: report of two cases. HSS J. 2007;3(2):17781.

8. Rathjen KE, Mubarak SJ. Calcaneal-cuboid-cuneiform osteotomy for the correction of valgus foot deformities in children. J Pediatr Orthop. 1998;18(6):775-82.

9. Davids JR, Gibson TW, Pugh LI. Quantitative segmental analysis of weight-bearing radiographs of the foot and ankle for children: normal alignment. J Pediatr Orthop. 2005; 25(6):769-76.
10. Kim JR, Shin SJ, Wang SI, Kang SM. Comparison of lateral opening wedge calcaneal osteotomy and medial calcaneal sliding-opening wedge cuboid-closing wedge cuneiform osteotomy for correction of planovalgus foot deformity in children. J Foot Ankle Surg. 2013;52(2):162-6.

11. Mosca VS. Calcaneal lengthening for valgus deformity of the hindfoot: results in children who had severe, symptomatic flatfoot and skewfoot. J Bone Joint Surg Am. 1995;77(4): 500-12.

12. Moraleda L, Mubarak SJ. Flexible flatfoot: differences in the relative alignment of each segment of the foot between symptomatic and asymptomatic patients. J Pediatr Orthop. 2011;31(4):421-8.

13. Kitaoka HB, Alexander IJ, Adelaar RS, Nunley JA, Myerson MS, Sanders M. Clinical rating systems for the ankle-hindfoot, midfoot, hallux, and lesser toes. Foot Ankle Int. 1994; 15(7):349-53.

14. Richter M, Zech S, Geerling J, Frink M, Knobloch K, Krettek C. A new foot and ankle outcome score: questionnaire based, subjective, visual-analogue-scale, validated and computerized. Foot Ankle Surg. 2006;12(4):191-9.

15. Westberry DE, Davids JR, Roush TF, Pugh LI. Qualitative versus quantitative radiographic analysis of foot deformities in children with hemiplegic cerebral palsy. J Pediatr Orthop. 2008;28(3):359-65.

16. Lee KM, Chung CY, Park MS, Lee SH, Cho JH, Choi IH. Reliability and validity of radiographic measurements in hindfoot varus and valgus. J Bone Joint Surg Am. 2010;92(13): 2319-27. 